Research Article

\title{
Peripapillary Microvascularization Analysis Using Swept-Source Optical Coherence Tomography Angiography in Optic Chiasmal Compression
}

\author{
Inès Ben Ghezala, ${ }^{1}$ Déa Haddad, ${ }^{1}$ Julie Blanc, ${ }^{1}$ Cyril Meillon, ${ }^{1}$ Rachid Madkouri, ${ }^{2}$ \\ François Borsotti, ${ }^{2}$ Alain M. Bron $\mathbb{D}^{1,},{ }^{1,3}$ and Catherine Creuzot-Garcher ${ }^{1,3}$ \\ ${ }^{1}$ Department of Ophthalmology, University Hospital, Dijon, France \\ ${ }^{2}$ Department of Neurosurgery, University Hospital, Dijon, France \\ ${ }^{3}$ Taste and Food Science Centre, AgroSup Dijon, CNRS, INRAE, Bourgogne Franche-Comté University, F-21000 Dijon, France
}

Correspondence should be addressed to Alain M. Bron; alain.bron@chu-dijon.fr

Received 28 January 2021; Revised 25 August 2021; Accepted 28 August 2021; Published 6 September 2021

Academic Editor: Sentaro Kusuhara

Copyright (c) 2021 Inès Ben Ghezala et al. This is an open access article distributed under the Creative Commons Attribution License, which permits unrestricted use, distribution, and reproduction in any medium, provided the original work is properly cited.

Purpose. To evaluate the vessel density (VD) of the radial peripapillary capillary (RPC) network using swept-source optical coherence tomography angiography (SS-OCTA) "en face" images of eyes with chiasmal compression caused by brain tumors before and after decompressive surgery compared with healthy controls. Methods. A cross-sectional study was conducted in 12 patients with chiasmal compression confirmed by neuroimaging. Sixteen healthy participants were also included. All patients with chiasmal compression underwent a neuro-ophthalmological examination one week before and 6 months after brain surgery, including static automated perimetry as well as measurement of the thickness of the retinal nerve fiber layer (RNFL) and the ganglion cell complex (GCC) with spectral-domain optical coherence tomography (SD-OCT). Based on this neuro-ophthalmological examination, the presence of an optic neuropathy $(\mathrm{ON})$ was evaluated. Peripapillary VD was obtained in four sectors on a $6 \times 6 \mathrm{~mm}$ SS-OCTA image using the Cirrus Plex Elite 9000. Results. Baseline average VD was significantly lower in patients with chiasmal compression and $\mathrm{ON}$ than in controls (median: 55.62; interquartile range (IQR): 2.96 vs. 58.53; IQR: 2.02; $p=0.003$ ). This decrease was also found in the temporal, superior, and nasal sectors. Average postoperative VD was decreased in patients with chiasmal compression compared with average preoperative VD (median: 56.16; IQR: 4.07 vs. 57.48; IQR: 3.83; $p=0.004$ ). Preoperative VD was significantly correlated with RNFL, GCC thickness, and visual field defects. Conclusions. The VD of the RPC network was decreased in chiasmal compressive $\mathrm{ON}$, and it was further decreased at 6 months after decompressive surgery.

\section{Introduction}

Various brain tumors can cause chiasmal compression: adenomas of the pituitary gland, craniopharyngiomas, pinealomas, Rathke cleft cysts, and meningiomas. Chiasmal compression predominantly affects the crossed nerve fibers associated with the nasal hemiretina, leaving uncrossed nerve fibers (coming from the temporal hemiretina) relatively well-preserved $[1,2]$. This compression increases the risk of damage to the axons of retinal ganglion cells, leading to deterioration of visual function, particularly of the visual field (VF). It is traditionally diagnosed by the presence of a characteristic bitemporal hemianopsia on the VF [3].

Optical coherence tomography (OCT) has been widely used to assess the morphological alterations of the retina and the peripapillary retinal nerve fiber layer (RNFL) in compressive optic neuropathy (ON). Previous studies have documented that peripapillary and macular retinal thickness, particularly RNFL, ganglion cell layer, and inner plexiform layer, were correlated with the location and the severity of the VF defects [4-7]. Nasal retinal thickness is especially strongly correlated with temporal VF [7]. 
Measurement of RNFL thickness was also used to predict visual recovery after decompressive surgery $[6,8,9]$. Macular ganglion cell complex (GCC) analysis has been shown to be especially useful in the early detection of chiasmal compression without VF defects, and it might be more sensitive than RNFL thickness [10-14].

Recent advances in optical coherence tomography angiography (OCTA) have led to the identification of several microvascular alterations in various ONs and retinal diseases. This recently developed imaging technique can noninvasively visualize the retinal and peripapillary microcirculation without dye injection $[15,16]$. Retinal vascularization is organized into four vascular plexuses, the superficial capillary plexus (SCP), which then anastomoses and generates the intermediate and deep capillary plexus (ICP and DCP, respectively) on either side of the inner nuclear layer, a superficial plexus in the RNFL around the optic nerve head, and the radial peripapillary capillary (RPC) plexus [17]. This RPC network cannot be clearly visualized with fluorescein angiography, unlike with OCTA images [18].

Recent studies have analyzed the contribution of OCTA in neuropathic disorders; microvascular changes were found in glaucoma [19-21], ischemic ON [22-24], and inflammatory ON [25-27]. A significant correlation was found between RPC density, RNFL thickness, and severity of visual function defects in glaucomatous eyes [20, 21]. Even in healthy eyes, a correlation between RNFL thickness and RPC density has been reported [28].

Nevertheless, to date, few studies have investigated the microvascular changes in eyes with chiasmal compression using OCTA [29-32]. Circumpapillary and macular vessel density have been shown to be lower in eyes with chiasmal compression than in healthy control eyes, especially in the nasal sectors [29, 31]. Circumpapillary and macular vessel density were correlated with RNFL thickness, GCC analysis, and VF defects [29].

These findings stimulated the hypothesis that a change in peripapillary microvascularization may be associated with a compressive $\mathrm{ON}$ and might be a predictive factor of postoperative VF recovery. Therefore, the present study aimed to quantify the VD of the RPC network using swept-source optical coherence tomography angiography (SS-OCTA) "en face" images from patients with chiasmal compression tumors and compare them with healthy control volunteers. We also evaluated the kinetics of VD after decompressive brain surgery and the correlation with other ocular metrics, such as VF defects, RNFL thickness, and GCC analysis.

\section{Materials and Methods}

2.1. Study Participants. We conducted a prospective study in the Department of Ophthalmology at the Dijon University Hospital (France) between April $1^{\text {st }}, 2019$, and March $1^{\text {st }}$, 2020. This study included consecutive patients with chiasmal compression confirmed by neuroimaging and healthy controls. The protocol was approved by the regional ethics committee and followed the tenets of the Declaration of Helsinki. All patients gave their written informed consent for the examination before enrolment. Patients were excluded if they had any retinal disease or optic nerve disease other than compressive ON. They were also excluded if they had a history of diabetes, cardiovascular diseases, or any other diseases that might affect the retina or the optic nerve. Concerning the brain lesion, patients were excluded if they had undergone any previous radiotherapy, surgery, or medical treatments. All patients had magnetic resonance imaging (MRI) of the brain that confirmed a lesion compressing the optic chiasm. All patients underwent decompressive brain surgery, a transsphenoidal tumor resection, at the Department of Neurosurgery of Dijon University Hospital. The control group consisted of healthy volunteers. Controls with a self-reported eye disease or with a history of cardiovascular or neurological disease were excluded.

2.2. Clinical Evaluation. All patients and healthy controls underwent a complete neuro-ophthalmological evaluation, including measurement of the best corrected visual acuity with a Monoyer chart, dilated fundoscopic examination, intraocular pressure measurement with a noncontact tonometer (Tonoref II, Nidek, Gamagori, Japan), globe axial length measurement using the IOL master 500 (Carl Zeiss Meditec, Jena, Germany), color vision testing with Ishihara plates, and VF and SD-OCT before and after surgery. The VF was assessed with an automated perimetry with the 30-2 Swedish interactive thresholding algorithm (SITA) standard strategy (Humphrey field analyzer, Carl Zeiss Meditec, Dublin, CA, USA). The reliability criteria were fixation loss of $\leq 20 \%$, false-positive results of $\leq 15 \%$, and false-negative results of $\leq 15 \%$. The mean deviation (MD) and pattern standard deviation (PSD) were used for the analysis. The SDOCT analysis was performed with the Cirrus HD-OCT 5000 (Carl Zeiss Meditec, Dublin, CA, USA). The RNFL thickness was determined using the $200 \times 200$ protocol optical disc cube with Cirrus software (Carl Zeiss Meditec, Dublin, CA, USA). This protocol provides an average thickness of the RNFL and the thickness in four sectors (temporal, superior, nasal, and inferior). For the GCC analysis, the thickness of the ganglion cell layer and the inner plexiform layer were automatically evaluated. Based on clinical examination, compatible VF defects, GCC, and RNFL loss on SD-OCT, we defined two subgroups of patients with chiasmal compression: patients with and without $\mathrm{ON}(\mathrm{ON}+$ and $\mathrm{ON}-$, respectively). Preoperative assessments were performed one week before surgery, and postoperative visits occurred 6 months after surgery. Figure 1 illustrates the multimodal analysis of a patient with chiasmal compression $\mathrm{ON}$ at the time of diagnosis.

2.3. OCTA Imaging. The SS-OCTA images were acquired with the Cirrus Plex Elite 9000 (Carl Zeiss Meditec, Jena, Germany), which uses a swept laser source with a central wavelength between $1040 \mathrm{~nm}$ and $1060 \mathrm{~nm}$ and a rate of 100,000 A-scans per second. For all study participants, the SS-OCTA examination was performed under mydriasis obtained with one drop of tropicamide $0.5 \%$ (Théa, Clermont-Ferrand, France). Patients underwent one imaging 
session including a $6 \times 6 \mathrm{~mm}$ scan centered on the optic nerve head. We used an active eye tracker to minimize motion artefacts. Eyes with poor signal strength $(<6 / 10)$ or significant artefacts on the perfusion map were excluded from the analysis.

The software automatically performed segmentation of the inner limiting membrane and the outer boundary of the RNFL to isolate the RPC vasculature "en face." Anonymized data files were downloaded from the Cirrus Plex Elite 9000 (Carl Zeiss Meditec, Jena, Germany) and uploaded to the advanced retina imaging (ARI) network portal. Image processing was performed with the peripapillary nerve fiber layer microvasculature density algorithm developed by Carl Zeiss Meditec (version 0.7).

2.4. Radial Peripapillary Capillary Analysis. Vessel density was defined as the percentage of the area occupied by vessels showing blood flow in a given region. The software automatically defined the center of the optic disc and identified four sectors (temporal, superior, nasal, and inferior) within two rings of $2 \mathrm{~mm}$ as the inner diameter and $6 \mathrm{~mm}$ as the outer diameter (Figure 1). Vessel density was provided in the four sectors as well as the average of these four sectors. The result was a percentage ranging from $0 \%$ (no perfusion) to $100 \%$ (fully perfused). The area occupied by large retinal vessels was not included in these calculations.

2.5. Statistical Analysis. Continuous variables were tested for normality (Shapiro-Wilk test). Continuous variables with a normal distribution are expressed as mean (standard deviation, $\mathrm{SD}$ ) and those with a nonnormal distribution are expressed as median (interquartile range, IQR). Categorical variables are summarized as numbers and percentages. Generalized estimating equation (GEE) regression models were used to consider intraindividual correlations between the two eyes to compare RNFL thickness, GCC thickness, visual field, and vessel density between patients and controls and between preoperative and postoperative values in patients. Spearman's correlation coefficients were used to determine the relationship between peripapillary VD and traditional metrics such as RNFL and GCC thickness and VF, PSD, and MD in eyes with chiasmal compression. A $p$ value of less than 0.05 was considered statistically significant. All statistical analyses were performed using SAS software (version 9.4; SAS Institute, Inc., Cary, NC, USA).

\section{Results}

The study included 14 consecutive patients with chiasmal compression and 16 healthy participants. Two patients with chiasmal compression were excluded owing to coexisting diabetes and multiple sclerosis. A total of 32 eyes from 16 healthy participants (group 1) and 24 eyes from 12 patients with chiasmal compression (group 2) were included in the analysis. Brain MRI revealed the following diagnoses: ten pituitary adenomas, one intrasellar arachnoid cyst, and one meningioma. An ON was found in 12 eyes from six patients (five pituitary adenomas and one meningioma). One eye of a patient with $\mathrm{ON}$ was excluded after neurosurgery due to poor SS-OCTA quality related to postoperative oculomotor paralysis, and the other eye was retained for analysis (Figure 2).

Tables 1 and 2 summarize the demographic as well as the structural and functional characteristics of the patients and healthy controls.

Table 3 presents the results of the comparison of VD across sectors between healthy controls and patients with chiasmal compression.

Baseline average VD was significantly lower in patients with chiasmal compression $\mathrm{ON}+$ than in controls $(p=0.003)$. Based on a sector comparison, VD was significantly reduced in patients with chiasmal compression $\mathrm{ON}+$ in the temporal, superior, and nasal sectors, compared with healthy controls $(p=0.005, p=0.02$, and $p=0.03$, respectively).

3.1. Vessel Density of the Radial Peripapillary Capillary 6 Months after Surgery. Table 4 lists the changes in VD in the RPC network 6 months after decompressive surgery in all sectors.

VD was reduced postoperatively compared with preoperatively in patients with chiasmal compression (median: 56.16; IQR: 4.07 vs. 57.48 ; IQR: 3.83 ; $p=0.004$ ). This significant reduction was also found in the temporal, superior, and inferior sectors.

3.2. Correlation between Vessel Density and Structural and Functional Metrics. Table 5 displays the Spearman correlation coefficients of VD before surgery with RNFL, GCC, and VF defects. The relationship between VD and RNFL thickness was analyzed in all patients with chiasmal compression in the different sectors segmented. A correlation was found between RNFL thickness and average, temporal, superior, and inferior VD $(p<0.001, p<0.001, p=0.004$, and $p=0.005$, respectively).

\section{Discussion}

In this study, we investigated peripapillary retinal microvascularization in patients with chiasmal compression before and after decompressive surgery and compared these patients with healthy volunteers. Average, temporal, superior, and nasal VD was significantly lower in patients with chiasmal compression $\mathrm{ON}+$ than in controls. Interestingly, VD was higher in patients with chiasmal compression ONthan in controls.

In the literature, it has been established that the vascular network plays a primary role in many ONs [27, 33]. However, the possibility of a primary vascular component in compressive ON is unlikely. The reduction in VD could be secondary to neural degeneration of the retina. It has already been established that chiasmal compression affects the nerve fibers in the nasal hemiretina $[1,2]$. This compression resulted in optic atrophy characterized clinically by band atrophy in the fundus [2], associated with a decrease in RNFL and GCC thicknesses [14, 34]. Functionally, it was 


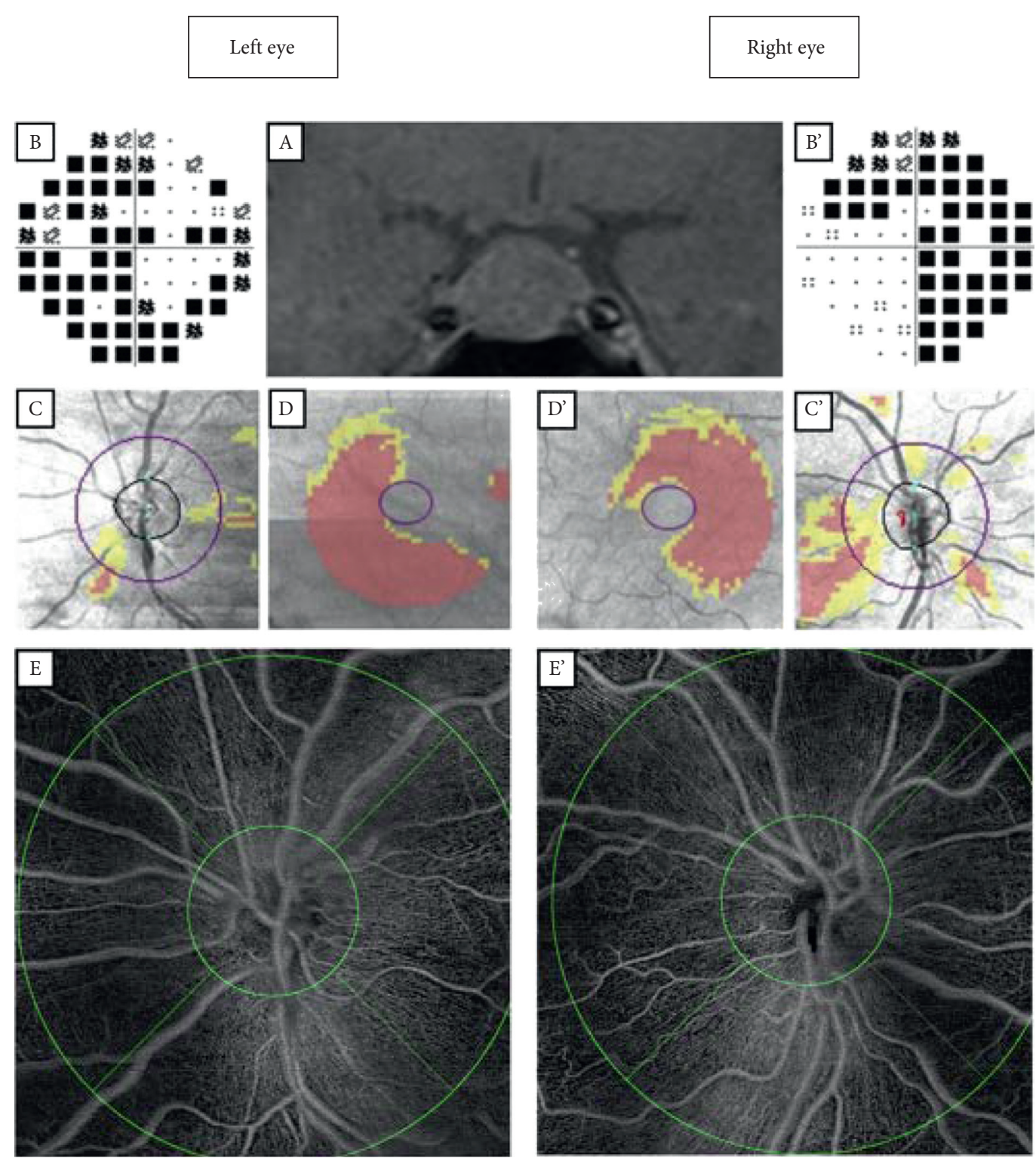

FIGURE 1: Multimodal analysis of a patient with a large pituitary adenoma with bilateral optic neuropathy. (A) Brain T1 magnetic resonance imaging showing a large pituitary adenoma with a mass effect on the optic chiasm. (B, $\left.B^{\prime}\right)$ Bitemporal hemianopsia on the Humphrey visual field (30-2). (C, $\left.C^{\prime}\right)$ Retinal nerve fiber layer thickness map. (D, D') Ganglion cell complex analysis. (E, E') "En face" optical coherence tomography angiography, $6 \times 6 \mathrm{~mm}$, with early treatment diabetic retinopathy study grid.

defined by bitemporal hemianopsia on the VF [3]. Therefore, the neuronal and axonal loss could reduce the metabolic activity in the inner layers of the retina, leading to a decrease in the demand for oxygen and blood with a constriction of precapillary arterioles [35]. Thus, this reduction could induce a regression of the superficial peripapillary capillary network, particularly pronounced in the nasal sector corresponding to axonal loss. This hypothesis of neurovascular metabolic feedback between loss of neurons and reduction in vascular perfusion has already been discussed but remains under debate $[31,36]$. However, the hypothesis appears to be consistent with our results since VD reduction was strongly correlated with RNFL thickness reduction.

We also investigated the changes in VD after decompressive surgery. The 6-month postoperative follow-up of patients demonstrated a significant decrease in average VD in patients with chiasmal compression $(p=0.004)$. We might have expected an increase in VD postoperatively after the removal of axonal compression, but this was not observed in the present case series, we found the opposite. Postoperative disc swelling may have gone undetected and could lead to changes in VD, as was previously described in other studies [37, 38].

Regarding the relationship between VD in SS-OCTA and other ocular metrics, a fairly positive correlation between average VD and RNFL thickness was found for patients with chiasmal compression $(r=0.69, p<0.001)$. This correlation was found with pre- and postoperative RNFL thickness and has already been shown in other studies on compressive ON [29] and in glaucomatous eyes [19]. 


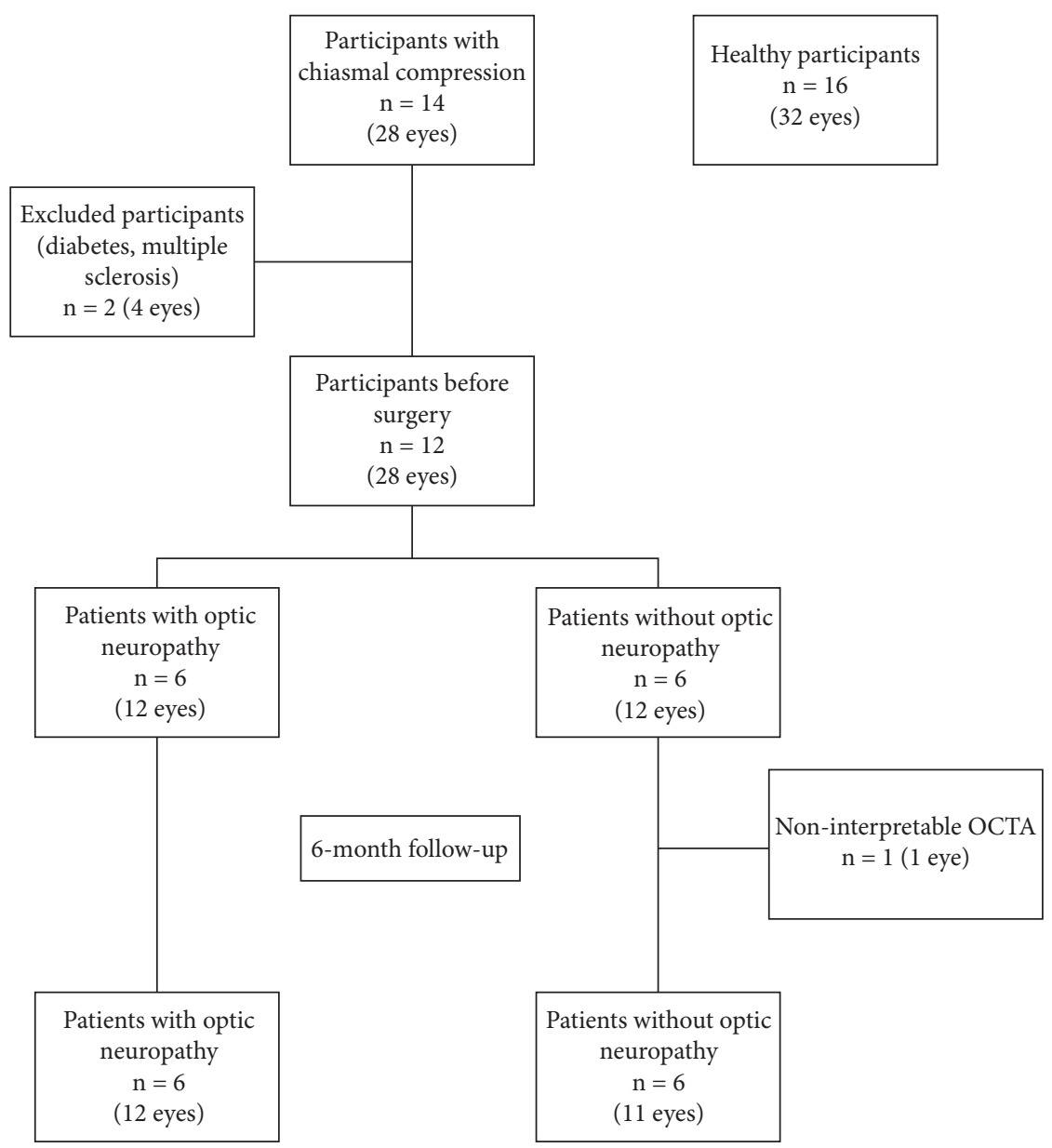

Figure 2: Flowchart of eligible and excluded patients and healthy participants.

TABLE 1: Demographic characteristics of study participants.

\begin{tabular}{lccc}
\hline & Healthy controls $(N=16)$ & Patients with chiasmal compression $(N=12)$ & $p$ value \\
\hline Eyes & 32 & 24 & $0.51^{*}$ \\
Sex, female & $13(81.2)$ & $53.1 \pm 14.5$ & $0.10^{* *}$ \\
Age, years & $46.7 \pm 14.1$ & $17.2 \pm 1.3$ & $0.11^{* *}$ \\
OOP, mmHG & $15.9 \pm 2.4$ & $23.9 \pm 1.2$ & $0.59^{* *}$ \\
Axial length, mm & $23.8 \pm 0.7$ & & \\
\hline
\end{tabular}

Continuous variables are displayed as mean \pm standard deviation. Categorical variables are displayed as number (percentage). IOP $=$ intraocular pressure.

${ }^{*}$ Fisher's exact test ${ }^{* *}$ Student's $t$-test.

Interestingly, we found a significant correlation between preoperative VD and preoperative VF, like Suzuki et al. [29]. Nevertheless, we did not find any correlation between preoperative VD and postoperative VF.

Our results are consistent with previous studies that have also demonstrated a decrease in peripapillary and macular VD in ON secondary to chiasmal compression [29]. Peripapillary and macular VD have been studied in patients with pituitary adenomas, and a significant decrease in VD in the peripapillary and macula SCP has been shown [39]. To our knowledge, only a few studies have investigated the impact of decompressive surgery on VD. Lee et al. also found a decrease in peripapillary VD after surgery [31]. One study found a correlation between preoperative peripapillary VD and postoperative $\mathrm{VF}$, suggesting this could be a prognostic factor [32]. In our study, although peripapillary VD decreased after surgery, patients had a relatively well-preserved postoperative VF (mean deviation $-2.8 \mathrm{~dB} \pm 5.5$ for patients who had chiasmal compressive $\mathrm{ON}$ ) and we did not find any correlation between average preoperative VD and postoperative VF ( $p=0.54$ for VF mean deviation). Interestingly, decreased retinal perfusion was also demonstrated after treatment of optic neuritis with good visual acuity recovery [40].

We acknowledge several limitations to this study. First, this is a small case series within a single center, which limits its external validity. Second, medical history was mostly selfreported, and we did not carry out any medical assessment 
TABLE 2: Structural and functional characteristics of patients with chiasmal compression.

\begin{tabular}{|c|c|c|c|c|c|c|}
\hline & \multicolumn{3}{|c|}{ Patients with chiasmal compression with $\mathrm{ON}$} & \multicolumn{3}{|c|}{ Patients with chiasmal compression without $\mathrm{ON}$} \\
\hline & $\begin{array}{c}\text { Preoperative }(n=6)(12 \\
\text { eyes })\end{array}$ & $\begin{array}{c}\text { Postoperative }(n=6)(12 \\
\text { eyes })\end{array}$ & $\begin{array}{c}p \\
\text { value }^{\dagger}\end{array}$ & $\begin{array}{c}\text { Preoperative }(n=6)(12 \\
\text { eyes })\end{array}$ & $\begin{array}{c}\text { Postop }(n=6)(11 \\
\text { eyes })\end{array}$ & $\underset{\text { value }^{\dagger}}{p}$ \\
\hline \multicolumn{7}{|l|}{ RNFL thickness, $\mu m^{*}$} \\
\hline Average & $76.4 \pm 2.3$ & $71.8 \pm 3.0$ & 0.001 & $90.7 \pm 2.6$ & $88.8 \pm 2.9$ & 0.42 \\
\hline Temporal & $48.8 \pm 2.4$ & $47.0 \pm 3.3$ & 0.06 & $66.8 \pm 4.3$ & $64.7 \pm 1.8$ & 0.52 \\
\hline Superior & $97.3 \pm 3.8$ & $88.0 \pm 4.8$ & $<0.001$ & $108.2 \pm 5.6$ & $105.7 \pm 6.3$ & 0.60 \\
\hline Nasal & $62.6 \pm 3.6$ & $58.0 \pm 2.1$ & 0.02 & $70.6 \pm 3.3$ & $70.5 \pm 3.9$ & 0.96 \\
\hline Inferior & $98.1 \pm 5.4$ & $94.4 \pm 7.3$ & 0.21 & $117.2 \pm 1.4$ & $117.1 \pm 2.3$ & 0.96 \\
\hline GCC thickness, $\mu m^{*}$ & & $63.8 \pm 3.3$ & & & & \\
\hline Average & $63.8 \pm 3.2$ & $63.8 \pm 3.3$ & 0.89 & $78.2 \pm 4.3$ & $77.1 \pm 3.1$ & 0.62 \\
\hline \multicolumn{7}{|l|}{ Visual field, $d B^{* *}$} \\
\hline Mean deviation & $-14.3 \pm 13.0$ & $-2.8 \pm 5.5$ & 0.003 & $-2.6 \pm 5.6$ & $-3.7 \pm 5.4$ & 0.32 \\
\hline $\begin{array}{l}\text { Pattern standard } \\
\text { deviation }\end{array}$ & $8.7 \pm 7.1$ & $3.4 \pm 7.2$ & 0.04 & $3.8 \pm 2.6$ & $3.0 \pm 3.1$ & 0.68 \\
\hline
\end{tabular}

${ }^{*}$ Variables are displayed as mean \pm standard deviation. ${ }^{* *}$ Variables are displayed as median \pm interquartile range. ${ }^{\dagger}$ Estimated using generalized estimating equation regression models. $\mathrm{ON}=$ optic neuropathy, RNFL = retinal nerve fiber layer, GCC = ganglion cell complex. No significant differences were found in gender and age between patients with chiasmal compression and healthy controls. No disc swelling was detected in the group of patients with chiasmal compression on fundus examination. In total, 13 eyes (54.2\%) had acquired dyschromatopsia (eight in the group of patients ON+). Vessel density of the radial peripapillary capillary before surgery. Bold values represent statistical significance.

TABLE 3: Vessel density comparison between healthy controls and patients with chiasmal compression with and without optic neuropathy.

\begin{tabular}{lcccc}
\hline & Healthy controls $(N=16)$ & \multicolumn{2}{c}{$\begin{array}{c}\text { Patients with chiasmal } \\
\text { compression with ON }(N=6)\end{array}$} & \multicolumn{2}{c}{$\begin{array}{c}\text { Patients with chiasmal } \\
\text { compression without ON }(N=6) \\
\text { Median } \pm \mathrm{IQR}\end{array}$} & $\begin{array}{c}\text { Median } \pm \mathrm{IQR} \\
p \text { value* }\end{array}$ & $\mathbf{0 . 0 0 3}$ & $59.44 \pm 1.95$ \\
\hline Average & $58.53 \pm 2.02$ & $55.62 \pm 2.96$ & $\mathbf{0 . 0 0 5}$ & $61.83 \pm 3.88$ \\
Temporal & $59.05 \pm 3.24$ & $56.12 \pm 3.63$ & $\mathbf{0 . 0 2}$ & $58.01 \pm 2.78$ \\
Superior & $57.91 \pm 3.99$ & $55.49 \pm 3.72$ & $\mathbf{0 . 0 3}$ & $57.12 \pm 2.80$ \\
Nasal & $56.49 \pm 3.62$ & $52.66 \pm 7.36$ & 0.15 & $61.08 \pm 4.02$ \\
Inferior & $59.73 \pm 4.20$ & $58.73 \pm 3.99$ & 0.001 \\
\hline
\end{tabular}

Variables are displayed as median \pm interquartile range. ${ }^{*}$ Estimated using generalized estimating equation regression models. ON $=$ optic neuropathy. $\mathrm{IQR}=$ interquartile range.

TABLE 4: Pre- and postoperative vessel density comparison of patients with chiasmal compression with and without optic neuropathy.

\begin{tabular}{lccc}
\hline & & Patients with chiasmal compression $(n=12)$ & \\
& Preoperative & $56.16 \pm 4.07$ & \\
\hline Average & $57.48 \pm 3.83$ & $57.59 \pm 6.95$ & $\mathbf{0 . 0 0 4}$ \\
Temporal & $58.30 \pm 5.71$ & $55.87 \pm 6.46$ & $\mathbf{0 . 0 2}$ \\
Superior & $57.02 \pm 2.94$ & $55.21 \pm 5.37$ & $\mathbf{0 . 0 3}$ \\
Nasal & $56.81 \pm 5.52$ & $58.70 \pm 4.40$ & 0.46 \\
Inferior & $59.94 \pm 4.54$ & $\mathbf{0 . 0 3}$ \\
\hline
\end{tabular}

Variables are displayed as median \pm interquartile range. ${ }^{*}$ Estimated using generalized estimating equation regression models. Bold values represent statistical significance.

or any blood tests to formally exclude vascular diseases in healthy participants. Third, we encountered artefacts with the acquisition of SS-OCTA images, for example, artefacts during OCTA image acquisition, including motion artefacts, and during image treatment such as automated segmentation failure [41]. However, projection artefacts were not expected to affect the measurements in this study given that the microvascularization within the RNFL is the most anterior retinal vascularization. Besides, only eyes with good SS-OCTA signal strength $(\geq 7 / 10)$ and without any significant artefact on the perfusion map were included. Fourth, the quantification grid was automatically centered on the optic disc for VD measurement. It is possible that this detection failed and resulted in inaccurate VD quantification. We therefore checked the correct location of the optic disc on the OCTA acquisitions. Fifth, we could not rule out the possibility of undetected microswelling in the optic disc affecting the assessment of VD. Sixth, automatic quantification using OCTA software has demonstrated good repeatability and reproducibility [42-44]. However, the repeatability and reproducibility of this measurement in SSOCTA with the Cirrus Plex Elite 9000 device (Carl Zeiss Meditec, Jena, Germany) have not been studied. Since the same measurements using different devices are not 
TABLE 5: Spearman's correlation coefficient $(r$ ( $p$ value $))$ of vessel density with pre- and postoperative structural and functional characteristics in patients with chiasmal compression.

\begin{tabular}{|c|c|c|c|c|c|}
\hline Preoperative vessel density & Average & Temporal & Superior & Nasal & Inferior \\
\hline \multicolumn{6}{|l|}{ Preoperative } \\
\hline RNFL thickness & $0.69(<\mathbf{0 . 0 0 1})$ & $0.75(<\mathbf{0 . 0 0 1})$ & $0.57(\mathbf{0 . 0 0 4})$ & $0.34(0.10)$ & $0.55(\mathbf{0 . 0 0 5})$ \\
\hline GCC thickness & $0.60(\mathbf{0 . 0 0 2})$ & NA & NA & NA & NA \\
\hline VF mean deviation & $0.64(\mathbf{0 . 0 0 1})$ & NA & NA & NA & NA \\
\hline VF pattern standard deviation & $-0.79(<\mathbf{0 . 0 0 1})$ & NA & NA & NA & NA \\
\hline \multicolumn{6}{|l|}{ Postoperative } \\
\hline RNFL thickness & $0.80(<\mathbf{0 . 0 0 1})$ & $0.80(<\mathbf{0 . 0 0 1})$ & $0.71(<\mathbf{0 . 0 0 1})$ & $0.61(\mathbf{0 . 0 0 2})$ & $0.55(\mathbf{0 . 0 0 6})$ \\
\hline GCC thickness & $0.58(\mathbf{0 . 0 0 3 )}$ & NA & NA & NA & NA \\
\hline VF mean deviation & $0.13(0.54)<$ & NA & NA & NA & NA \\
\hline VF pattern standard deviation & $-0.38(0.07)$ & NA & NA & NA & NA \\
\hline
\end{tabular}

$\mathrm{RNFL}=$ retinal nerve fiber layer, $\mathrm{GCC}=$ ganglion cell complex, $\mathrm{VF}=$ visual field, $\mathrm{NA}=$ not applicable.

comparable, further study should evaluate the repeatability and reproducibility of this measurement with the Cirrus Plex Elite 9000 device (Carl Zeiss Meditec, Jena, Germany) [45].

In conclusion, the VD of the RPC network was decreased in chiasmal compressive ON. This decrease was correlated with other optic nerve metrics. The effect of decompressive surgery may potentially play a role in induced and additional decrease in the final VD reduction.

\section{Data Availability}

Datasets analyzed during the current study are available from the corresponding author upon request.

\section{Ethical Approval}

All procedures performed in studies involving human participants were in accordance with the ethics standards of the institutional and/or national research committee (Dijon Ethics committee) and with the 1964 Declaration of Helsinki and its later amendments or comparable ethical standards.

\section{Conflicts of Interest}

Catherine Creuzot-Garcher is a consultant for Allergan, Bayer, Horus, Novartis, Roche, and Théa. Alain M. Bron is a consultant for Aerie, Allergan, Bausch-Lomb, Santen Pharmaceutical, and Théa. The other authors declare no conflicts of interest.

\section{References}

[1] W. F. Hoyt and O. Luis, "The primate chiasm," Archives of Ophthalmology, vol. 70, no. 1, pp. 69-85, 1963.

[2] R. Unsöld and W. F. Hoyt, "Band atrophy of the optic nerve," Archives of Ophthalmology, vol. 98, no. 9, pp. 1637-1638, 1980.

[3] M. Grochowicki, A. Vighetto, S. Berquet, Y. Khalfallah, and G. Sassolas, "Pituitary adenomas: automatic static perimetry and Goldmann perimetry. A comparative study of 345 visual field charts," British Journal of Ophthalmology, vol. 75, no. 4, pp. 219-221, 1991.

[4] A. Kanamori, M. Nakamura, N. Matsui et al., "Optical coherence tomography detects characteristic retinal nerve fiber layer thickness corresponding to band atrophy of the optic discs," Ophthalmology, vol. 111, no. 12, pp. 2278-2283, 2004.
[5] R. B. D. Araújo, M. K. Oyamada, L. C. Zacharias, L. P. Cunha, R. C. Preti, and M. L. R. Monteiro, "Morphological and functional inner and outer retinal layer abnormalities in eyes with permanent temporal hemianopia from chiasmal compression," Frontiers in Neurology, vol. 8, p. 619, 2017.

[6] H. V. Danesh-Meyer, A. Wong, T. Papchenko et al., "Optical coherence tomography predicts visual outcome for pituitary tumors," Journal of Clinical Neuroscience, vol. 22, no. 7, pp. 1098-1104, 2015.

[7] M. L. R. Monteiro, K. Hokazono, D. B. Fernandes et al., "Evaluation of inner retinal layers in eyes with temporal hemianopic visual loss from chiasmal compression using optical coherence tomography," Investigative Opthalmology \& Visual Science, vol. 55, no. 5, pp. 3328-3336, 2014.

[8] Y. Yoneoka, T. Hatase, N. Watanabe et al., "Early morphological recovery of the optic chiasm is associated with excellent visual outcome in patients with compressive chiasmal syndrome caused by pituitary tumors," Neurological Research, vol. 37, no. 1, pp. 1-8, 2015.

[9] M. T. M. Wang, J. King, R. C. A. Symons et al., "Prognostic utility of optical coherence tomography for long-term visual recovery following pituitary tumor surgery," American Journal of Ophthalmology, vol. 218, pp. 247-254, 2020.

[10] R. J. Blanch, J. A. Micieli, N. M. Oyesiku, N. J. Newman, and V. Biousse, "Optical coherence tomography retinal ganglion cell complex analysis for the detection of early chiasmal compression," Pituitary, vol. 21, no. 5, pp. 515-523, 2018.

[11] H. R. Yum, S. H. Park, H.-Y. L. Park, and S. Y. Shin, "Macular ganglion cell analysis determined by cirrus HD optical coherence tomography for early detecting chiasmal compression," PLoS One, vol. 11, no. 4, Article ID e0153064, 2016.

[12] G. Orman, G. Sungur, and C. Culha, "Assessment of inner retina layers thickness values in eyes with pituitary tumours before visual field defects occur," Eye, vol. 35, no. 4, pp. 1159-1164, 2021.

[13] M. G. Tieger, T. R. Hedges, J. Ho et al., "Ganglion cell complex loss in chiasmal compression by brain tumors," Journal of Neuro-Ophthalmology, vol. 37, no. 1, pp. 7-12, 2017.

[14] A. Akashi, A. Kanamori, K. Ueda, Y. Matsumoto, Y. Yamada, and M. Nakamura, "The detection of macular analysis by SDOCT for optic chiasmal compression neuropathy and nasotemporal overlap," Investigative Opthalmology \& Visual Science, vol. 55, no. 7, pp. 4667-4672, 2014.

[15] P. J. Rosenfeld, M. K Durbin, L. Roisman et al., "ZEISS Angioplex $^{\mathrm{TM}}$ spectral domain optical coherence tomography angiography: technical aspects," Developments in Ophthalmology, vol. 56, pp. 18-29, 2016. 
[16] R. F. Spaide, J. G. Fujimoto, N. K. Waheed, S. R. Sadda, and G. Staurenghi, "Optical coherence tomography angiography," Progress in Retinal and Eye Research, vol. 64, pp. 1-55, 2018.

[17] J. P. Campbell, M. Zhang, T. S. Hwang et al., "Detailed vascular anatomy of the human retina by projection-resolved optical coherence tomography angiography," Scientific Reports, vol. 7, no. 1, p. 42201, 2017.

[18] R. F. Spaide, J. M. Klancnik Jr., and M. J. Cooney, "Retinal vascular layers imaged by fluorescein angiography and optical coherence tomography angiography," JAMA Ophthalmology, vol. 133, no. 1, pp. 45-50, 2015.

[19] L. Liu, Y. Jia, H. L. Takusagawa et al., "Optical coherence tomography angiography of the peripapillary retina in glaucoma," JAMA Ophthalmology, vol. 133, no. 9, pp. 1045-1052, 2015.

[20] Z. Mammo, M. Heisler, C. Balaratnasingam et al., "Quantitative optical coherence tomography angiography of radial peripapillary capillaries in glaucoma, glaucoma suspect, and normal eyes," American Journal of Ophthalmology, vol. 170, pp. 41-49, 2016.

[21] A. Yarmohammadi, L. M. Zangwill, A. Diniz-Filho et al., "Relationship between optical coherence tomography angiography vessel density and severity of visual field loss in glaucoma," Ophthalmology, vol. 123, no. 12, pp. 2498-2508, 2016.

[22] E. Augstburger, P. Zéboulon, C. Keilani, C. Baudouin, and A. Labbé, "Retinal and choroidal microvasculature in nonarteritic anterior ischemic optic neuropathy: an optical coherence tomography angiography study," Investigative Opthalmology \& Visual Science, vol. 59, no. 2, pp. 870-877, 2018.

[23] E. D. Gaier, M. Wang, A. L. Gilbert, J. F. Rizzo, D. M. Cestari, and J. B. Miller, "Quantitative analysis of optical coherence tomographic angiography (OCT-A) in patients with nonarteritic anterior ischemic optic neuropathy (NAION) corresponds to visual function," PLoS One, vol. 13, no. 6, Article ID e0199793, 2018.

[24] E. Wright Mayes, E. D. Cole, S. Dang et al., "Optical coherence tomography angiography in nonarteritic anterior ischemic optic neuropathy," Journal of Neuro-Ophthalmology, vol. 37, no. 4, pp. 358-364, 2017.

[25] N. Feucht, M. Maier, G. Lepennetier et al., "Optical coherence tomography angiography indicates associations of the retinal vascular network and disease activity in multiple sclerosis," Multiple Sclerosis Journal, vol. 25, no. 2, pp. 224-234, 2019.

[26] W. R. Kwapong, C. Peng, Z. He, X. Zhuang, M. Shen, and F. Lu, "Altered macular microvasculature in neuromyelitis optica spectrum disorders," American Journal of Ophthalmology, vol. 192, pp. 47-55, 2018.

[27] X. Wang, Y. Jia, R. Spain et al., "Optical coherence tomography angiography of optic nerve head and parafovea in multiple sclerosis," British Journal of Ophthalmology, vol. 98, no. 10, pp. 1368-1373, 2014.

[28] X. Ding, L. Lu, J. Yang, Y. Chen, and J. Ma, “The peripapillary retinal capillary density is highly correlated with its nerve fibre layer in normal population," Clinical Hemorheology and Microcirculation, vol. 74, no. 3, pp. 231-239, 2020.

[29] A. C. F. Suzuki, L. C. Zacharias, R. C. Preti, L. P. Cunha, and M. L. R. Monteiro, "Circumpapillary and macular vessel density assessment by optical coherence tomography angiography in eyes with temporal hemianopia from chiasmal compression. Correlation with retinal neural and visual field loss," Eye, vol. 34, no. 4, pp. 695-703, 2020.
[30] E. J. Lee, J.-A. Kim, T.-W. Kim, H. Kim, H. K. Yang, and J.-M. Hwang, "Glaucoma-like parapapillary choroidal microvasculature dropout in patients with compressive optic neuropathy," Ophthalmology, vol. 127, no. 12, pp. 1652-1662, 2020.

[31] G.-I. Lee, K.-A. Park, S. Y. Oh, and D.-S. Kong, "Analysis of optic chiasmal compression caused by brain tumors using optical coherence tomography angiography," Scientific Reports, vol. 10, no. 1, p. 2088, 2020.

[32] G.-I. Lee, K.-A. Park, S. Y. Oh, and D.-S. Kong, "Parafoveal and peripapillary perfusion predict visual field recovery in chiasmal compression due to pituitary tumors," Journal of Clinical Medicine, vol. 9, no. 3, p. 697, 2020.

[33] K. Sakaguchi, T. Higashide, S. Udagawa, S. Ohkubo, and K. Sugiyama, "Comparison of sectoral structure-function relationships in glaucoma: vessel density versus thickness in the peripapillary retinal nerve fiber layer," Investigative Opthalmology \& Visual Science, vol. 58, no. 12, pp. 5251-5262, 2017.

[34] C. H. Moon, S. H. Lee, B.-T. Kim, S. C. Hwang, Y.-H. Ohn, and T. K. Park, "Diagnostic ability of retinal nerve fiber layer thickness measurements and neurologic hemifield test to detect chiasmal compression," Investigative Opthalmology \& Visual Science, vol. 53, no. 9, pp. 5410-5415, 2012.

[35] J. Sebag, F. C. Delori, G. T. Feke, and J. J. Weiter, "Effects of optic atrophy on retinal blood flow and oxygen saturation in humans," Archives of Ophthalmology, vol. 107, no. 2, pp. 222-226, 1989.

[36] R. Parrozzani, F. Leonardi, L. Frizziero et al., "Retinal vascular and neural remodeling secondary to optic nerve axonal degeneration," Ophthalmology Retina, vol. 2, no. 8, pp. 827-835, 2018.

[37] M. A. Fard, J. Jalili, A. Sahraiyan et al., "Optical coherence tomography angiography in optic disc swelling," American Journal of Ophthalmology, vol. 191, pp. 116-123, 2018.

[38] F. Tuntas Bilen and H. Atilla, "Peripapillary vessel density measured by optical coherence tomography angiography in idiopathic intracranial hypertension," Journal of NeuroOphthalmology, vol. 39, no. 3, pp. 319-323, 2019.

[39] L. Dallorto, C. Lavia, A.-L. Jeannerot et al., "Retinal microvasculature in pituitary adenoma patients: is optical coherence tomography angiography useful?" Acta Ophthalmologica, vol. 98, pp. 585-592, 2019.

[40] T. Higashiyama, Y. Nishida, and M. Ohji, "Optical coherence tomography angiography in eyes with good visual acuity recovery after treatment for optic neuritis," PLoS One, vol. 12, no. 2, Article ID e0172168, 2017.

[41] R. F. Spaide, J. G. Fujimoto, and N. K. Waheed, "Image artifacts in optical coherence tomography angiography," Retina, vol. 35, no. 11, pp. 2163-2180, 2015.

[42] M. Al-Sheikh, T. C. Tepelus, T. Nazikyan, and S. R. Sadda, "Repeatability of automated vessel density measurements using optical coherence tomography angiography," British Journal of Ophthalmology, vol. 101, no. 4, pp. 449-452, 2017.

[43] F. Coscas, A. Sellam, A. Glacet-Bernard et al., "Normative data for vascular density in superficial and deep capillary plexuses of healthy adults assessed by optical coherence tomography angiography," Investigative Opthalmology \& Visual Science, vol. 57, no. 9, 2016.

[44] J. I. Fernández-Vigo, B. Kudsieh, A. Macarro-Merino et al., "Reproducibility of macular and optic nerve head vessel density measurements by swept-source optical coherence tomography angiography," European Journal of Ophthalmology, vol. 30, no. 4, pp. 756-763, 2020. 
[45] F. Corvi, M. Pellegrini, S. Erba, M. Cozzi, G. Staurenghi, and A. Giani, "Reproducibility of vessel density, fractal dimension, and foveal avascular zone using 7 different optical coherence tomography angiography devices," American Journal of Ophthalmology, vol. 186, pp. 25-31, 2018. 